\title{
Médiévales
}

Langues, Textes, Histoire

70 | printemps 2016

Lieux d'hygiène et lieux d'aisance en terre d'Islam (VII $\mathrm{e}_{-}$ $\mathrm{XV}^{\mathrm{e}}$ siècle)

\section{Trois latrines publiques dans la Jérash de l'Antiquité tardive (Jordanie)}

Three Public Latrines in Late Antique Jerash (Jordan)

\section{Louise Blanke}

Traducteur : Marie-Odile Rousset

\section{OpenEdition}

\section{Journals}

Édition électronique

URL : http://journals.openedition.org/medievales/7763

DOI : 10.4000/medievales.7763

ISSN : 1777-5892

\section{Éditeur}

Presses universitaires de Vincennes

\section{Édition imprimée}

Date de publication : 23 juin 2016

Pagination : 43-58

ISSN : 0751-2708

\section{Référence électronique}

Louise Blanke, «Trois latrines publiques dans la Jérash de l'Antiquité tardive (Jordanie) », Médiévales [En ligne], 70 | printemps 2016, mis en ligne le 15 juin 2018, consulté le 21 avril 2019. URL : http:// journals.openedition.org/medievales/7763 ; DOI : 10.4000/medievales.7763 


\section{Louise Blanke}

\section{Trois latrines publiques dans la Jérash de l'Antiquité tardive (Jordanie)}

La ville de Jérash, dans le nord de la Jordanie, a été fouillée extensivement au siècle dernier ${ }^{1}$. Des projets, tel celui de la Yale Joint Mission dans les années 1920 et $1930^{2}$, le Jerash Archaeological Project dans les années $1980^{3}$, et des fouilles en cours effectuées par le Département des Antiquités jordaniennes, ont transformé le paysage et font de ce site archéologique l'un des mieux connus de Jordanie. Ces interventions ont mis au jour une architecture monumentale, principalement des périodes romaine et byzantine, incluant des temples, des théâtres, des bains et des églises alignés sur un réseau de rues à colonnades ${ }^{4}$. L'intérêt privilégié porté jusqu'alors aux vestiges les plus monumentaux a fait que les archéologues n'ont commencé à explorer le passé islamique de Jérash que récemment, élargissant ainsi notre compréhension de l'histoire de la ville de plusieurs siècles $^{5}$; mais l'histoire de la vie quotidienne à Jérash durant cette période

1. Je suis très reconnaissante à Marie-Odile Rousset, Patrice Cressier et Sophie Gilotte de m'avoir invitée à participer à ce dossier de Médiévales. Un merci sincère va également à Stefanie Hoss et Luke Treadwell qui ont lu les versions préliminaires de ce texte et fourni des commentaires éclairés sur son contenu. Merci également à Alan Walmsley, à l'équipe de l'Islamic Jerash Project, au Département des Antiquités de Jordanie, sans lesquels cet article n'aurait pas été écrit, à Daniel Reynolds, Don Boyer et Stephen McPhillips qui ont fourni des commentaires importants et à Thomas Lepaon, qui nous a autorisés à reproduire la figure 1 .

2. L'intitulé Yale Joint Mission fait référence aux fouilles entreprises par l'université de Yale et la British School of Archaeology de Jerusalem (1928-1930) et à la collaboration entre l'Université de Yale et l'American School of Oriental Research (1930-1931, 1933-1934).

3. F. ZAYADINE éd., Jarash Archaeological Project 1981-1983, Amman, 1986.

4. C. H. Kraeling éd., Gerasa, City of the Decapolis, New Haven, 1938.

5. Il s'agit du projet jordano-danois Islamic Jerash Project (voir les références n. 21), du projet germano-danois Jerash Northwest Quarter Project (voir, par exemple, A. Lichtenberger et R. RAJA, " Towards an Understanding of the Dark Ages of Islamic Jerash. Connecting the Evidence through Texts and Archaeology », Comparative Islamic Studies, à paraître) et du Late Antique Jerash Project (voir L. BlanKe, H. BARNES, 
et celles qui lui sont antérieures demeure encore largement méconnue. Cependant, la présence de trois latrines publiques découvertes durant ces enquêtes éclaire certains des aspects les plus banals de la vie urbaine.

Une des latrines est localisée sur la place centrale de la ville, à l'intersection de deux rues principales - le cardo et le decumanus sud et deux ont été trouvées à l'entrée de bains (fig. 1). Les études menées au cœur de l'empire romain, en Italie, révèlent que des latrines publiques étaient le plus souvent implantées dans ou à côté des bains ou près de points d'eau à usage collectif comme des fontaines, où elles pouvaient bénéficier de l'approvisionnement en eau préexistant et du système d'évacuation ${ }^{6}$. La forme prise par ces installations est le plus souvent celle de banquettes en bois ou en pierre avec des ouvertures en forme de trou de serrure, disposées au-dessus d'un égout et sur les trois côtés d'une pièce. La capacité de ces latrines varie considérablement, mais elles peuvent, en général, accueillir douze à quinze utilisateurs en même temps ${ }^{7}$. L'eau douce était disponible dans une gouttière devant les sièges pour le nettoyage des installations après utilisation.

L'apparition de latrines publiques au Proche-Orient est intimement liée à l'extension de l'Empire romain. Dans un premier temps, elles furent introduites dans les camps militaires ou dans les villes avec une forte présence romaine, puis elles sont devenues au cours des siècles partie intégrante du tissu urbain de nombreux grands centres comme Apamée et Scythopolis. Néanmoins, la lecture des rapports archéologiques suggère qu'elles ont été trouvées le plus souvent au cœur de l'empire romain, tandis que leur introduction au Proche-Orient aurait été plus lente et qu'elles y sont plus rares ${ }^{8}$. Les informations publiées ont conduit Zena Kamash à conclure que nombre de villes n'avaient pas de latrines publiques et que celles-ci n'étaient habituellement pas associées aux bains'. Utiliser une latrine publique de style romain relevait d'une expérience vraiment communautaire : il n'y avait aucun écran ou compartiment pour préserver

M. BrondgaArd Jensen, R. Rattenborg et E. Thing, « The 2011 Season of the Late Antique Jarash Project : Results from the Survey Southwest of the Umayyad Congregational Mosque », Annual of the Department of Antiquities of Jordan, 57 (2013), p. 229-238). Voir aussi les études dans F. ZAYADINE éd., Jarash Archaeological...

6. J. VAN VAERENBERGH, "Flush Water for Toilets in and near the Baths », dans C. G. Jansen, A. O. Koloski-Ostrow et E. M. Moormann éd., Roman Toilets : Their Archaeology and Cultural History, Louvain, 2011, p. 78-86 ; J. VAN VAERENBERGH, « Location of Toilets within Baths », dans Ibid., p. 115-119.

7. Z. KAMASH, "Which Way to Look ? Latrine use in the Roman World », dans H. Molotch et L. NORÉn éd., Toilets : Public Restrooms and the Politics of Sharing, New York, 2010, p. 47-64 (p. 49).

8. Ibid., p. 59.

9. Ibid. 


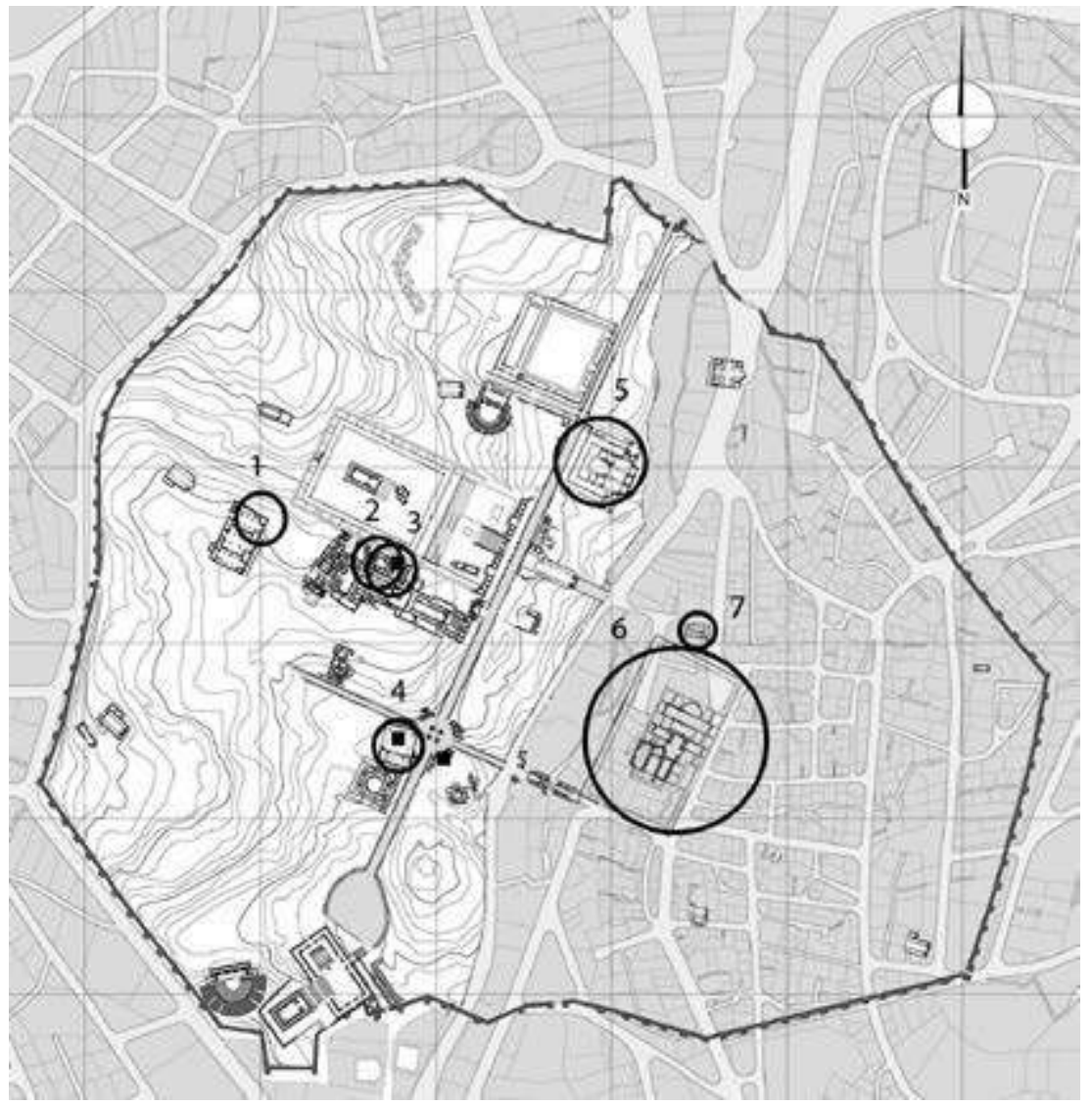

Fig. 1. Plan de Jérash avec localisation des bains (dans les cercles) et des trois latrines publiques (carrés) présentées dans cet article

1. Bains dans l'église désaffectée des Saints-Côme-et-Damien ; 2. Bains de la « Glass Court »; 3. Bains de Placcus ; 4. Bains centraux ; 5. Bains ouest ; 6. Bains est ; 7. Bains byzantins.

\section{(Adapté de T. LEPAON, « Un nouveau plan pour Jarash/Gerasa (Jordanie)»,} Annual of the Department of Antiquities of Jordan, 55 (2011), fig. 1)

l'intimité ou protéger les sens olfactifs ou auditifs ${ }^{10}$. Ces conditions, et la quasi-absence des latrines dans les données archéologiques du ProcheOrient, ont incité Warwick Ball à suggérer que «perhaps the only difference between baths in the East and the West were that the former never included communal public lavatories ${ }^{11}$. Il considère l'usage des toilettes au ProcheOrient comme restreint à la sphère privée et suggère que le caractère public

10. Ibid., p. 50.

11. W. BALL, Rome in the East. The Transformation of an Empire, Londres/New York, 2000, p. 304. 
des latrines était considéré comme inacceptable dans la vie urbaine de la région.

La présence à Jérash de trois de celles-ci, datant de la période romaine et de l'Antiquité tardive, remet en cause ces hypothèses. Cet article soutient que l'absence supposée de latrines dans le tissu urbain du Proche-Orient romain et de l'Antiquité tardive est le résultat de l'attention exclusive que les archéologues ont accordée aux vestiges architecturaux monumentaux, ainsi que du caractère partiel des publications. Dans le cas des bains, les fouilleurs se sont traditionnellement attachés aux espaces destinés à la toilette, tandis que les structures auxiliaires (latrines et zones de service) ont été largement ignorées, tout comme les systèmes d'alimentation en eau et d'évacuation. Les avancées récentes en archéologie, combinées avec un intérêt croissant pour la vie sociale des populations anciennes, ont encouragé l'étude des latrines, et de nouvelles analyses ont commencé à révéler les riches données qui peuvent être extraites de celle des bâtiments. Ces travaux prennent non seulement en compte ce que ces découvertes peuvent apporter à propos de la vie au-dessus des égouts, mais ils considèrent également l'archéobotanique et l'étude des anciennes maladies comme partie intégrante de l'examen des latrines $^{12}$.

Sept installations balnéaires ont été identifiées à Jérash (fig. 1). Il s'agit des bains monumentaux indépendants est et ouest, des bains plus petits de Placcus, des bains centraux, des bains byzantins, des bains dans la « Glass Court » (une cour dans laquelle a été retrouvée une grande quantité de verre cassé) et des bains dans l'église désaffectée des Saints-Côme-etDamien ${ }^{13}$. Parmi eux, seuls ceux de Placcus et les bains centraux ont été substantiellement fouillés et tous deux contiennent des latrines. Le troisième exemple pris en compte ici est situé en bordure de la place publique, à l'intersection de deux des rues les plus actives de la ville.

Le compte rendu qui suit présente tour à tour les trois latrines découvertes à Jérash entre 2008 et 2010, dans le cadre du projet jordanodanois Islamic Jerash Project, selon la chronologie de leur construction. L'étude examine leur disposition, leur approvisionnement en eau et leur système d'évacuation et, par comparaison avec des installations trouvées ailleurs au Proche-Orient, elle se propose d'évaluer le nombre de leurs utilisateurs. Enfin, nous verrons ce que ces latrines peuvent nous enseigner de la vie quotidienne dans l'ancienne Jérash.

12. Par exemple, P. D. Mitchell éd., Sanitation, Latrines and Intestinal Parasites in Past Populations, Farnham, 2015.

13. J. W. Crowford, « The Christian Churches », dans C. H. Kraeling éd., Gerasa..., p. 171-264 (p. 246-248). Merci à Daniel Reynolds pour m'avoir indiqué cette référence. 


\section{Une latrine dans le quart sud-est de la place du tétrapyle}

À Jérash, le croisement du cardo et du decumanus sud est marqué par une large place circulaire de 43,60 m de diamètre avec, au centre, un tétrapyle. Sur ses bords sont alignées de petites pièces, qui ont été interprétées comme des boutiques ${ }^{14}$. La place a été modifiée et son apparence actuelle remonte au IV $\mathrm{IV}^{\mathrm{e}}$ siècle $^{15}$. Avant cette nouvelle configuration, les rues se croisaient à angle droit et les travaux archéologiques suggèrent que, dans le rectangle qu'elles forment au sud-est, furent installées des latrines publiques ${ }^{16}$. Elles ont été fouillées et publiées par la Yale Joint Mission ${ }^{17}$. Une évacuation, délimitée par des tuiles en céramique et de près d'un mètre de profondeur, court le long des quatre murs extérieurs (fig. 2). La canalisation s'étend sur $13 \mathrm{~m} \mathrm{du}$ nord au sud et sur $12 \mathrm{~m}$ d'est en ouest et mesure 0,40 m de large. Elle a été coupée au nord-ouest par la construction de la façade circulaire de la place qui n'a laissé que $3 \mathrm{~m}$ des deux parties subsistantes. Bien qu'aucun siège n'ait été retrouvé, un rebord dans les murs est et sud indique leur emplacement. La canalisation s'incline doucement vers l'ouest, suggérant que l'évacuation s'écoulait dans l'égout en-dessous du cardo.

La publication de Carl Hermann Kraeling n'offre pas d'indication sur la façon dont les latrines étaient nettoyées mais on peut supposer qu'une source d'eau existait à proximité. Les travaux récents de Don Boyer montrent que la principale alimentation en eau du secteur occidental de Jérash provenait d'un aqueduc au nord-ouest du mur de l'enceinte urbaine. Sa capacité maximale était de $45000 \mathrm{~m}^{3}$ par jour ${ }^{18}$. L'eau était conduite à travers le mur jusqu'à de grands réservoirs creusés dans la roche, sur les collines derrière le temple d'Artemis ${ }^{19}$ et dans le quart sud-ouest de la ville ${ }^{20}$. De là, elle était distribuée par gravité aux quartiers situés en contrebas. Une source alternative d'alimentation en eau de la moitié ouest de la cité antique venait du réservoir de Birketein, à $2 \mathrm{~km}$ au nord de Jérash. Une série de fontaines, le long du côté ouest du cardo, parmi lesquelles le

14. C. H. Kraeling, « III. The South Tetrapylon », dans C. H. Kraeling éd., Gerasa..., p. $103-115$ (p. 105).

15. W. THIEL, « Tetrakionia. Überlegungen zu einem Denkmaltypus Tetrarchischer Zeit im Osten des Römischen Reiches », Antiquité Tardive, 10 (2002), p. 299-326.

16. C. H. KraEling, « III. The South Tetrapylon », dans C. H. KRAELING éd., Gerasa..., p. 113 .

17. Ibid.

18. Don Boyer, communication personnelle, février 2015.

19. A. Lichtenberger, A. Lindross, R. RAjA et J. Heinemeier, « Radiocarbon Analysis of Mortar from Roman and Byzantine Water Management Installations in the Northwest Quarter of Jerash, Jordan », Journal of Archaeological Science : Reports, 2 (2015), p. 114-127.

20. L. BLANKE et al., « The 2011 Season of the Late Antique Jarash Project... ». 


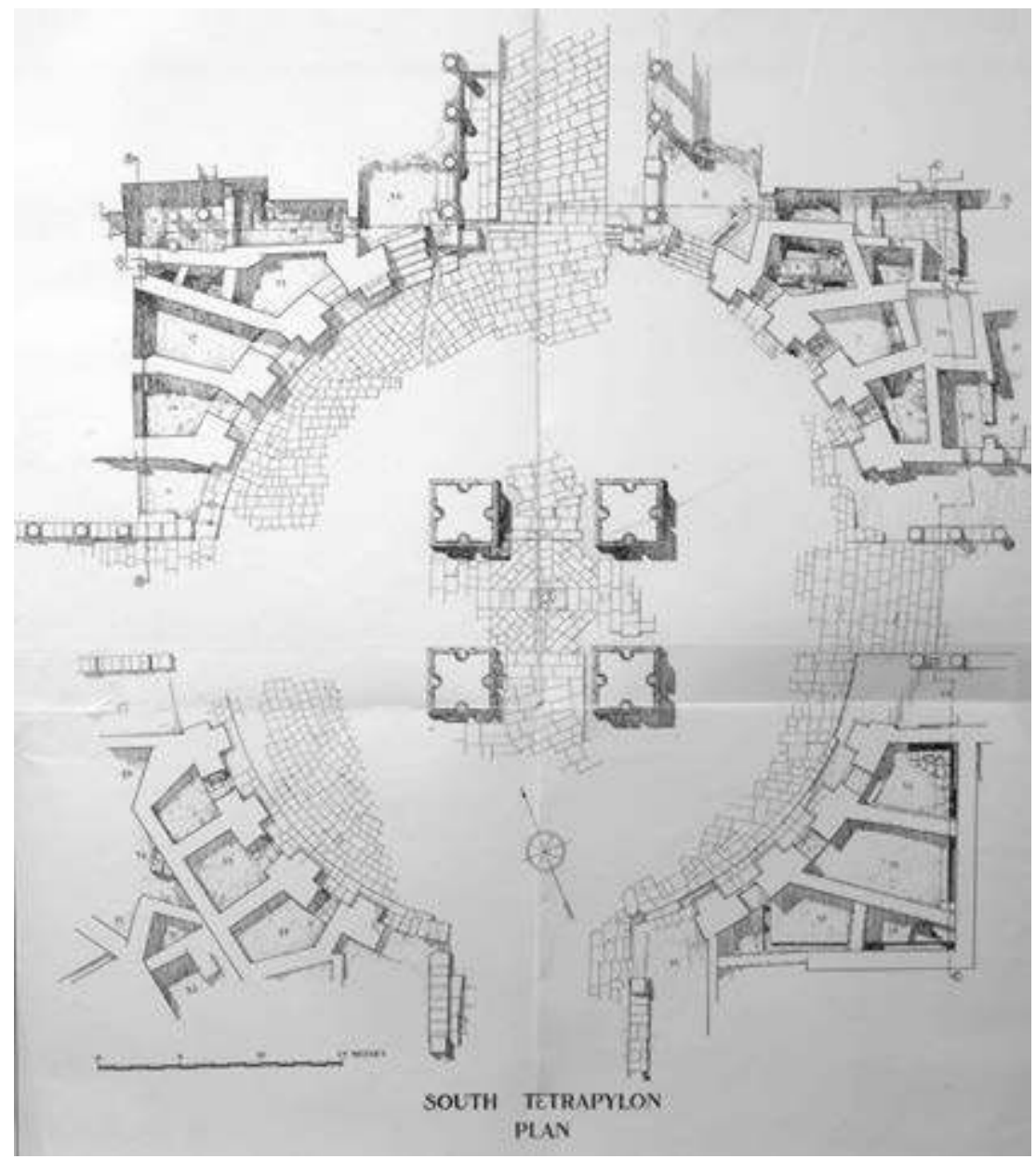

Fig. 2. Plan de la place à l'intersection du cardo et du decumanus sud Noter le drain dans le quart sud-est.

(D'après C. H. Kraeling éd., Gerasa, City of the Decapolis, New Haven, 1938, plan XII)

nymphaeum, montrent que l'eau était disponible en abondance et qu'une partie pouvait être employée au nettoyage des latrines de la place.

Le mobilier retrouvé dans la canalisation inclut une grande quantité de tessons de céramique et quelques monnaies datant, parmi d'autres, de Julia Mamaea (180-235 ap. J.-C.), Constantin (272-337 ap. J.-C.) et Constance. La publication ne mentionne pas de quel Constance il s'agit, mais vu la fourchette de dates des autres découvertes monétaires, une datation du IV siècle est probable. 


\section{Les latrines des bains centraux}

Les bains centraux sont localisés dans le centre du quartier commercial de Jérash, dans l'angle sud-ouest de l'intersection du cardo et du decumanus sud. Ils bordent le macellum au sud et la place du tétrapyle au nord-est. Les bains ont été identifiés pour la première fois en 1998 par une équipe du département jordanien des Antiquités qui fouillait deux zones tests dans lesquelles ont été partiellement découvertes des salles chaudes. La fouille de ces bains s'est poursuivie de 2002 à 2010 dans le cadre du projet jordano-danois Islamic Jerash Project (dorénavant IJP) ${ }^{21}$. Les bains ont été intégrés dans le projet IJP comme élément de l'enquête générale sur le développement de ce secteur de la ville pendant l'Antiquité tardive et la haute époque islamique. Les témoignages céramologiques et numismatiques suggèrent qu'ils furent construits à la fin $\mathrm{du} \mathrm{III}^{\mathrm{e}} \mathrm{ou}$ au $\mathrm{IV}^{\mathrm{e}}$ siècle ap. J.-C. et désaffectés dans la première partie du VIII ${ }^{\mathrm{e}}$ siècle. Utilisés en continu sur une période de 350 ans, ils sont passés par au moins cinq phases architecturales majeures. Ils ont été démantelés durant le processus de réaménagement de la zone pour la construction d'une mosquée congrégationnelle omeyyade, ce qui signifie que les données archéologiques proviennent presque exclusivement des fondations et des débris issus de la démolition et modification du bâtiment, avec assez peu de superstructure conservée.

Les bains centraux n'étaient pas monumentaux et indépendants comme les complexes balnéaires occidental et oriental, mais ils étaient incorporés au tissu urbain et intégraient plusieurs composantes architecturales (fig. 3). À côté des pièces d'eau, ils consistaient en une zone de service, des boutiques, une ou deux pièces de déshabillage et des latrines. Les boutiques étaient situées au nord des bains proprement dits, flanquant le

21. Le projet Islamic Jerash Project est dirigé par Alan Walmsley et j'ai été chargée de fouiller et publier les bains. Les anciennes publications sur les bains incluent : H. BARNES, L. Blanke, K. Damgaard, I. Simpson, M. L. Soerensen et A. Walmsley, « From 'Guard House' to Congregational Mosque : Recent Discoveries on the Urban History of Islamic Jarash », Annual of the Department of Antiquities of Jordan, 50 (2006), p. 285-314 ; L. Blanke, P. D. Lorien, R. RattenborG, "Changing Cityscapes in Central Jarash Between Late Antiquity and the Abbasid Period », Annual of the Department of Antiquities of Jordan, 54 (2010), p. 311-327 ; L. BLANKE et A. WALMSLEY, « The Islamic Jarash Project », dans D. R. Keller et C. A. TUTTLE éd., « Archaeology in Jordan, 2008 and 2009 seasons », American Journal of Archaeology, 114 (2010), p. 505-545 ; L. BLANKE et A. WALMSLEY, «The Islamic Jarash Project», dans D. R. Keller, B. A. Porter et C. A. TUTTLE éd., « Archaeology in Jordan, 2010 and 2011 Seasons », American Journal of Archaeology, 116 (2012), p. 693-750 ; A. WALMSLEY et K. DAMGAARD, « The Umayyad Congregational Mosque of Jarash in Jordan and its Relationship to Early Mosques », Antiquity, 79 (2005), p. 363-378 ; A. Walmsley, L. Blanke, K. Damgaard, A. Mellah, S. McPhillips, L. RoEnJE, I. Simpson et F. BesSARD, « A Mosque, Shops and Bath in Central Jarash : The 2007 Season of the Islamic Jarash Project », Annual of the Department of Antiquities of Jordan, 52 (2008), p. 109-137. 


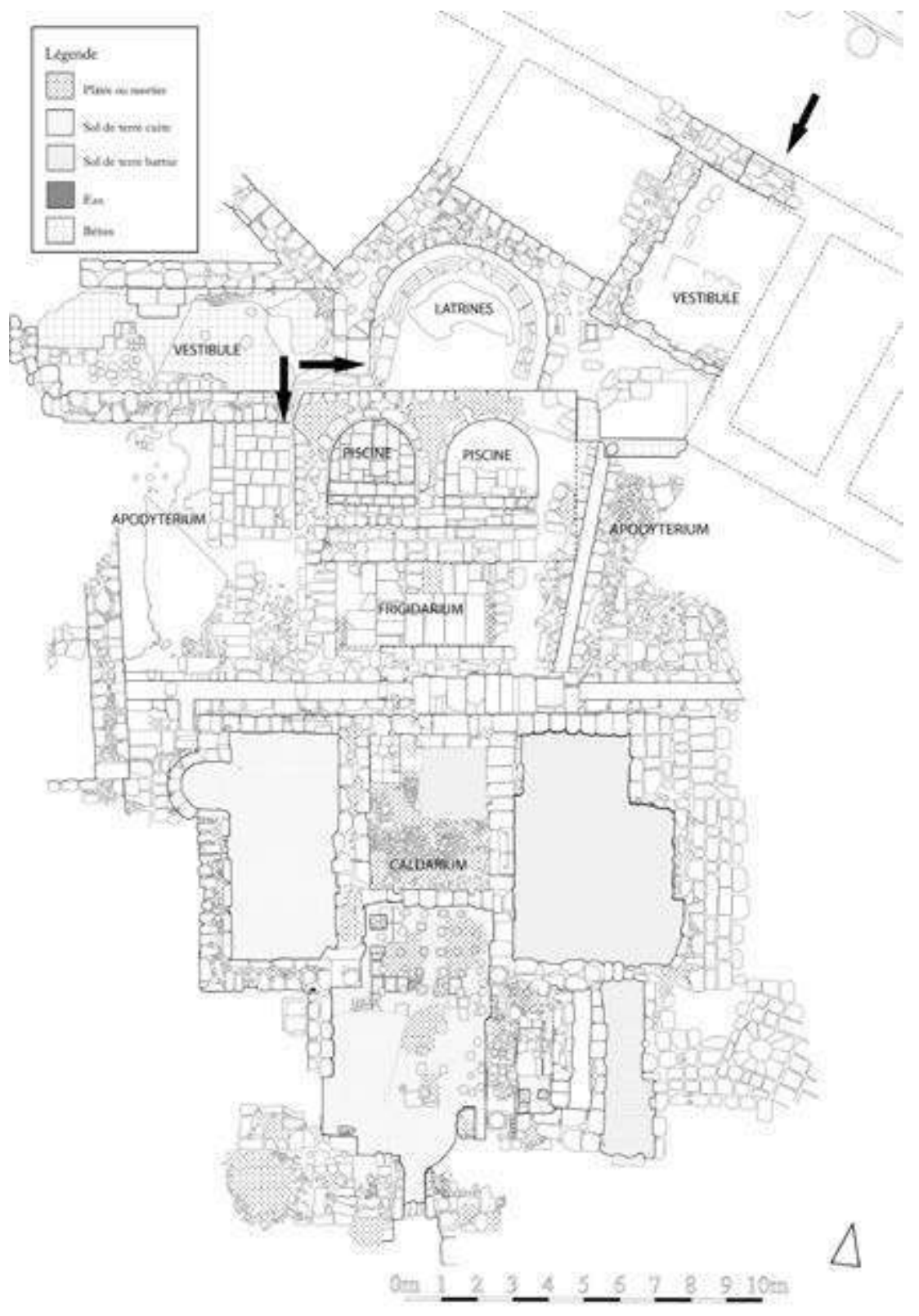

Fig. 3. Plan des bains centraux

Les flèches indiquent les entrées.

(C) Louise Blanke) 
decumanus sud, et peut-être aussi le long du cardo, de l'autre côté de la rue du macellum, bien que ces deux dernières zones n'aient pas été explorées par des fouilles.

Il était possible d'accéder aux bains par deux entrées différentes. Une porte entre deux boutiques du decumanus donnait accès à une petite pièce à partir de laquelle un vestiaire - un apodyterium - pouvait être atteint. Un second point d'entrée a été trouvé à l'extrémité occidentale des bains, dans une plus petite rue parallèle au decumanus. Il ouvrait sur un petit hall avec deux portes - l'une conduisant au sud à un second apodyterium et l'autre à l'est à des latrines. De cette manière, les visiteurs pouvaient utiliser cellesci sans nécessairement payer le droit d'entrée aux bains.

Les latrines ont été fouillées durant deux saisons en 2008 et 2010. La première campagne a permis de découvrir la structure elle-même, tandis que la seconde s'est centrée sur l'égout et l'étude préliminaire du matériel associé. Les latrines sont structurellement intégrées au plan des bains, ce qui sous-entend qu'elles ont été en usage pendant la durée de vie des bains centraux. Situées au nord des pièces d'eau, entre les boutiques et les bains eux-mêmes, elles adoptent une forme très inhabituelle, au plan semicirculaire ou plutôt en fer à cheval, au lieu du quadrilatère, plus fréquent (fig. 4). L'explication de ce choix pourrait se trouver dans ses qualités fonctionnelles plutôt que dans des considérations esthétiques - je reviendrai sur ce point plus loin.

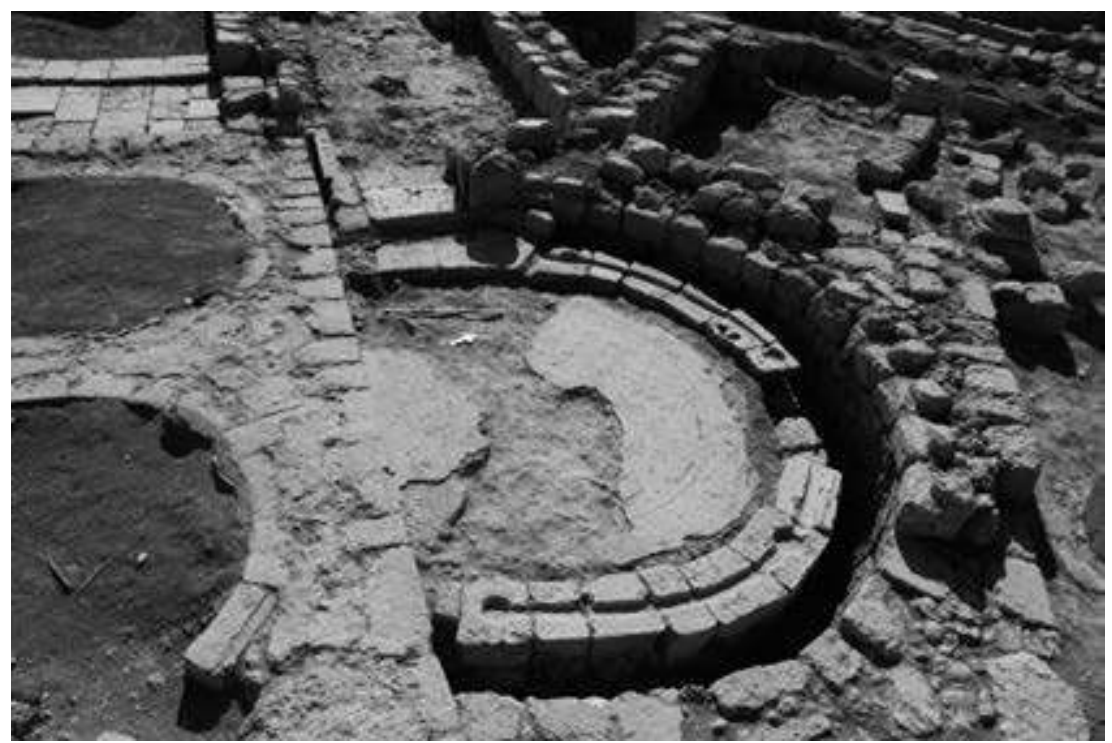

Fig. 4. Vue d'ensemble des latrines à l'extrémité nord des bains centraux (C) Louise Blanke) 
Les latrines ont été identifiées par leurs caractéristiques distinctives. Un égout court sur toute la longueur du mur nord incurvé. Il mesure $0,50 \mathrm{~m}$ de large et $1,30 \mathrm{~m}$ de profondeur avec une pente du sud-est vers le sudouest et a été construit avec des blocs de pierre, des tuiles et de plus petites pierres placées au fond. Son sol et ses parois étaient grossièrement enduits d'un mortier d'opus signinum. Aucun reste de siège n'a été retrouvé, mais un rebord dans le mur de pierre nord indique leur emplacement d'origine. Le sol de la pièce était recouvert d'une mosaïque avec un motif simple en rouge et blanc. Une gouttière part de l'angle sud-ouest de la pièce et suit toute la longueur de l'égout dans lequel elle disparaît dans l'angle sud-est. Cette gouttière était alimentée par un tuyau encastré dans le mur sud des latrines, qui recevait l'eau propre de la même source que les bassins du frigidarium adjacent. La gouttière peu profonde $(5 \mathrm{~cm}$ de profondeur et $10 \mathrm{~cm}$ de largeur seulement) recevait très probablement une alimentation en eau constante durant les heures d'ouverture des bains, ce qui devait prévenir la stagnation des urines et des fèces et réduire ainsi les odeurs ${ }^{22}$. Le plan était organisé de telle manière que les deux bassins semi-circulaires du frigidarium s'évacuaient dans l'égout des latrines, démontrant qu'elles étaient nettoyées régulièrement. La quantité de trouvailles, comme la céramique, suggère cependant que le flux n'était pas suffisamment puissant pour chasser complètement le matériel accumulé dans l'égout. Depuis les latrines, une évacuation se dirigeait vers le decumanus où les eaux usées rejoignaient un des collecteurs principaux de la ville.

L'examen de ces latrines a inclus une reconnaissance complète de l'égout, qui a donné lieu à une grande quantité de découvertes, particulièrement dans les dépôts des niveaux les plus profonds. Ces matériaux ne faisaient pas partie du remblai mis en place à l'abandon des latrines, mais se sont accumulés alors que l'égout était encore en usage, jetés et pour d'autres perdus dans les latrines. Ce mobilier est, par conséquent, un reflet direct des activités qui avaient lieu dans les bains centraux et offre un aperçu unique de la vie quotidienne dans des bains de l'Antiquité tardive. Ils comprennent différentes sortes de bijoux (perles, bracelets, anneaux et boucles d'oreilles), mais aussi des pièces de jeux et de grandes quantités d'os animaux, de verre et de céramique. Les fouilles ont produit pas moins de $80 \mathrm{~kg}$ de céramique dont la majorité appartient à des coupes à vin, d'après l'interprétation de Stephen McPhillips, ainsi que des flacons à huile et à parfum. Les céramiques ont toutes été datées des $\mathrm{VI}^{\mathrm{e}}$ et VII ${ }^{\mathrm{e}}$ siècles, laissant supposer que l'égout était à l'occasion nettoyé car il ne contenait pas de matériel contemporain de son utilisation ancienne. L'étude des découvertes dans les latrines est en cours et sera publiée dans un article

22. G. C. M. JANSEN, "Waste Disposal from Toilets », dans G. C. M. JANSEN, A. O. Koloski-Ostrow et E. M. MoORmann éd., Roman Toilets..., p. 76-78 (p. 76). 
qui associera études de matériel et analyses des restes archéobotaniques ${ }^{23}$. La localisation des latrines entre une rangée de boutiques et le bain luimême laisse supposer que la pièce était éclairée artificiellement - hypothèse confirmée par la découverte dans l'égout de plusieurs lampes à huile ${ }^{24}$.

Le plan particulier des bains et, notamment, la forme des latrines résultent de la réponse architecturale à une situation où deux réseaux urbains se rejoignent à l'intérieur d'un même bâtiment. Les boutiques à l'extrémité nord du complexe balnéaire suivent l'orientation du réseau défini par les rues. Cependant, à l'époque de la construction de ces dernières, un autre plan urbain existait déjà. Les bains mêmes montrent, en effet, un décalage d'environ $30^{\circ}$ par rapport au tracé de la rue, indiquant que des bâtiments existaient sans doute déjà à l'ouest, avec lesquels les bains devaient être en partie alignés. Les deux réseaux ont été combinés dans le complexe thermal par une série de solutions architecturales ingénieuses dont la plus remarquable se trouve être les latrines. Plutôt que de respecter un plan parfaitement semi-circulaire, la partie orientale de la pièce est légèrement décalée vers le centre. La disposition du mur, partagé par les latrines et les boutiques au nord, facilite la jonction de deux angles : l'extrémité occidentale du massif mesure 1,80 m de large et son extrémité orientale seulement 1,20 m. La combinaison de latrines semi-circulaires et de ce mur a permis d'utiliser et de camoufler une partie du bâtiment dont la forme originelle est trapézoïdale.

La proximité physique entre les latrines des bains centraux et celles du quart sud-est de la place du tétrapyle est importante. Les bains centraux ont été construits à peu près au moment où la place a été remodelée et devraient peut-être être considérés comme un élément d'une plus grande reconfiguration du centre de Jérash. Les latrines de la place auraient été désaffectées à ce moment, signifiant qu'une installation a remplacé l'autre. Une explication alternative serait que les deux latrines publiques aient été utilisées simultanément à un moment du $\mathrm{IV}^{\mathrm{e}}$ siècle. Dans tous les cas, la proximité des deux latrines, leur localisation dans des zones occupées et facilement accessibles, leur taille et l'usage continu des latrines des bains centraux jusqu'au VII ${ }^{\mathrm{e}}$ siècle suggèrent qu'elles étaient une composante importante de la vie urbaine à Jérash durant l'Antiquité tardive.

23. Un article collectif sera publié par l'auteur avec Stephen McPhillips et Pernille Bangsgaard Jensen.

24. Communication personnelle de Stephen McPhillips. 


\section{Les latrines des bains de Placcus}

Les troisièmes latrines ont été trouvées dans les bains de l'évêque Placcus pendant les fouilles de la Yale Joint Mission ${ }^{25}$. Ces bains sont situés entre les murs extérieurs du temple d'Artemis et l'église de Saint-Théodore. Leur entrée principale dnnait sur une ruelle en escalier. Leur attribution à l'évêque Placcus a été facilitée par une inscription commémorative sur une architrave de l'atrium, qui révèle également que le bâtiment a été construit en 454-455 ap. J.-C. D'après une seconde inscription, une restauration aurait eu lieu en $584^{26}$.

On pénétrait dans le bâtiment par un long portique (fig. 5, B34), qui donnait accès à une antichambre avec deux pièces, celle de droite étant les latrines (fig. 5, B35). Celles-ci et l'antichambre étaient séparées des bains mêmes par une autre porte, ce qui signifie qu'il était possible de fermer ces derniers tout en laissant un accès aux latrines. De plus, cet emplacement sous-entend que les latrines n'étaient pas seulement fréquentées par les baigneurs mais aussi par des non-baigneurs qui n'étaient ainsi pas obligés de payer les frais d'entrée.

Les latrines n'ont été décrites que très brièvement ${ }^{27}$. D'après la publication de Clarence S. Fisher, il est possible de déduire qu'elles mesuraient environ $5 \mathrm{~m}$ sur $3 \mathrm{~m}$. Elles ont été identifiées par la présence d'une rangée de pierres en forme de « $U$ » avec une cannelure centrale profonde, qui pourrait avoir soutenu les blocs dressés sur lesquels le bord des sièges aurait reposé. Un égout circulait derrière cet aménagement, mais on ne saisit pas, à la lecture de la publication, si l'égout a été fouillé en même temps que le reste des latrines par la Yale Joint Mission. Thomas Lepaon a récemment réexaminé les bains de Placcus et, dans le cadre de cette étude, il a (re)fouillé les égouts des latrines. Les résultats de ces travaux paraîtront prochainement dans la publication de son doctorat sur les thermes de Jérash.

D'après Fisher, les latrines étaient nettoyées avec l'eau de la citerne des bains, qui se situait dans la zone de service ${ }^{28}$ (fig. 5, B43). Un tuyau en céramique conduisait l'eau du réservoir par la zone de service jusqu'à l'égout, tandis qu'un second tuyau remplissait les bassins du caldarium. Il parait surprenant que les latrines aient été nettoyées avec de l'eau propre plutôt que celle du caldarium. La comparaison de ce dispositif avec celui des latrines des bains centraux permet de supposer que l'eau propre était plutôt utilisée dans la gouttière qui court en avant des sièges, tandis que les

25. C. S. Fisher, « Buildings of the Christian Period », dans C. H. KraELING éd., Gerasa..., p. 265-296.

26. Ibid., p. 265.

27. Ibid., p. 266.

28. Ibid., p. 268. 


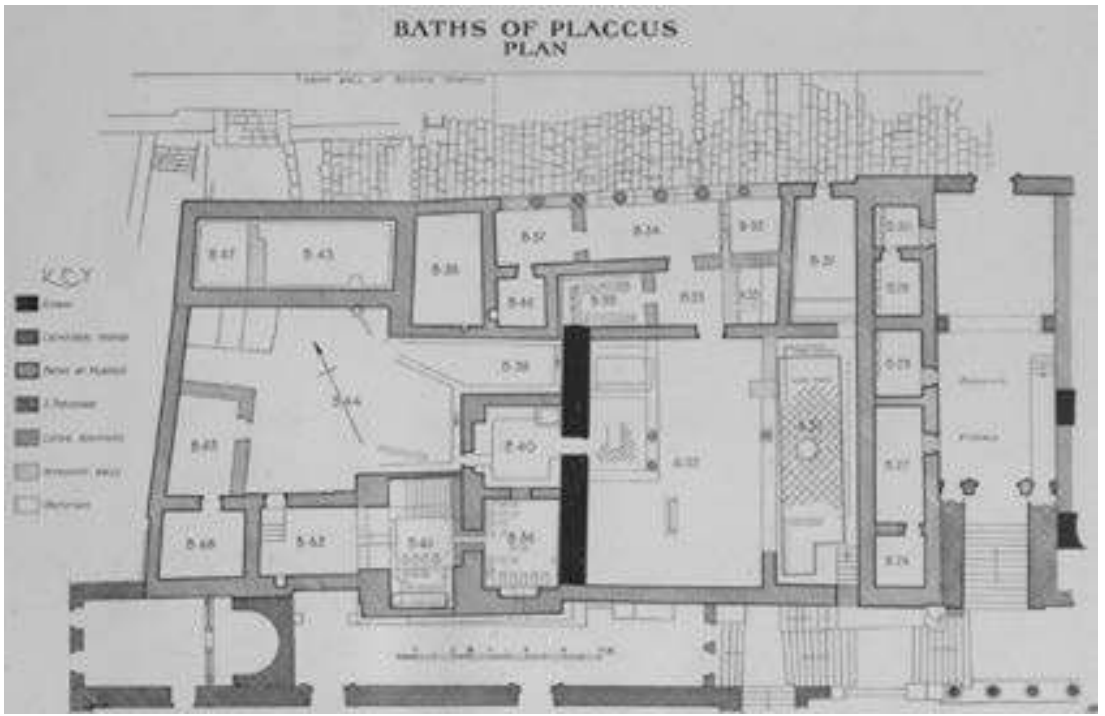

Fig. 5. Bains de Placcus

(D'après C. H. Kraeling éd., Gerasa, City of the Decapolis, New Haven, 1938, plan XLIV)

eaux usées, provenant des évacuations des bassins, ont pu être employées pour nettoyer les égouts. Le fait que les fouilles aient été concentrées sur les superstructures des bains et non sur leurs infrastructures pourrait expliquer que Fisher n'ait pas pris en compte une telle interconnexion.

La publication de Fisher sur les bains de Placcus n'offre aucun commentaire sur le système d'évacuation des bains. Pourtant, si l'on considère leur localisation sur un versant de colline, l'eau devait logiquement s'évacuer vers le cardo, alimentant ainsi le principal système collecteur de la ville.

\section{Discussion et remarques conclusives}

Les trois latrines identifiées jusqu'ici parmiles vestiges archéologiques de Jérash sont localisées dans des secteurs de la ville qui étaient accessibles au public : à l'intersection de deux des rues les plus fréquentées et à proximité de l'entrée de deux édifices de bains. Ces localisations étaient idéales en termes d'alimentation et d'évacuation en eau. Bien qu'aucun siège n'ait été retrouvé dans l'une ou l'autre de ces installations, il est possible d'estimer leur capacité maximale. Les études sur les latrines, dans d'autres parties du monde romain, ont révélé que la distance entre les sièges peut beaucoup varier : ils pouvaient être placés jusqu'à $0,90 \mathrm{~m}$ de distance ou plus les 
uns des autres ${ }^{29}$. Considérant la profondeur d'une chaise moderne (environ $0,50 \mathrm{~m}$ ), une distance confortable entre les sièges se situerait autour de 0,65 m. Si l'on applique cette distance aux latrines de Jérash, celles des bains centraux pouvaient recevoir treize utilisateurs, celles des bains de Placcus quinze et celles de la place du tétrapyle pouvaient accueillir le nombre impressionnant de quarante-cinq personnes. Bien qu'hypothétique, il est aussi possible de suggérer une capacité journalière maximale. En supposant une durée de fonctionnement de douze heures par jour, à raison d'environ un quart d'heure par usager, pour prendre en compte le temps pendant lequel les sièges restaient inoccupés, les latrines des bains centraux pouvaient servir à soulager 624 usagers par jour, les bains de Placcus 720 usagers, et les latrines de la place pouvaient recevoir jusqu'à 2160 visites journalières. Ce chiffre considérable et le fait que la construction des bains centraux comprenait des latrines à un moment où celles de la place ont été abandonnées donnent à penser que ces installations publiques étaient une composante importante de la vie quotidienne dans la ville.

La localisation des latrines ne suggère pas seulement qui pouvait utiliser ces installations, mais aussi quand elles étaient ouvertes au public. Celles des bains centraux et celles des bains de Placcus étaient situées près de l'entrée des complexes balnéaires. Les portes séparant les latrines des entrées réelles des bains indiquent qu'elles pouvaient être utilisées en dehors des horaires d'ouverture des édifices balnéaires. Cependant, une autre série de portes permettait aux deux latrines d'être complètement fermées au public, ce qui implique qu'elles n'étaient sans doute pas ouvertes 24 heures par jour (fig. 5, au nord de B33 ; fig. 6). Les trouvailles de fragments de lampes dans l'égout des bains centraux ne nous aident pas à comprendre les horaires d'ouverture. Comme cela a déjà été mentionné, leur présence peut indiquer un usage nocturne, mais résulte plus probablement d'une lumière naturelle insuffisante. L'accès aux latrines de la place est moins bien compris car rien ne subsiste de la façade du bâtiment.

L'importante quantité de tessons de céramique, verre, os, monnaies et petits objets retrouvés dans les égouts des latrines nous fournit non seulement un aperçu unique sur la vie des personnes qui utilisaient cellesci et les bains avoisinants, mais révèle aussi des aspects de l'usage des latrines elles-mêmes. La comparaison entre les matériaux exhumés dans les égouts des bains centraux et ceux des autres latrines semble indiquer que la principale différence ne réside pas dans le type de matériel mais dans sa taille. Les dimensions des tessons et des os d'animaux dans l'égout des latrines montrent qu'ils ne pouvaient pas avoir été évacués par les

29. S. Hoss, « Case Study : Rotttenburg Latrine », dans G. C. M. JANSEN, A. O. KolOSKIOstrow et E. M. MOORMAnN éd., Roman Toilets...,p. 67 ; A. O. KolOSKI-Ostrow, « Design and Architecture », dans Ibid., p. 51-55, (p. 53). 


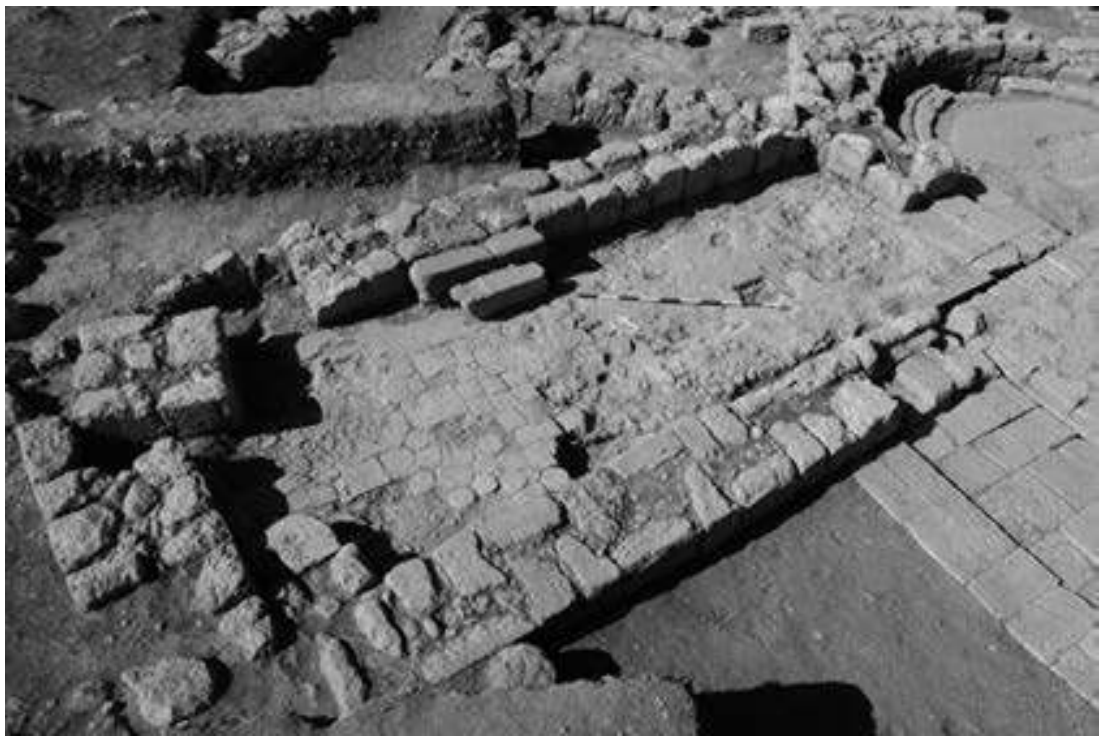

Fig. 6. Entrée des bains centraux et des latrines

Les escaliers conduisent de l'espace extérieur au hall d'entrée. Noter deux portes à l'extrémité orientale.

(C) Louise Blanke)

canalisations des bains. Les latrines pouvaient donc avoir une utilisation secondaire comme moyen facile de se débarrasser de déchets.

Des études collectives récentes ont éclairé la question de la fonction et de l'utilisation des latrines dans la partie occidentale du monde romain ${ }^{30}$ mais elles restent à conduire pour la partie orientale de l'Empire afin d'identifier et de publier des latrines urbaines au Proche-Orient. La première étape passe par une révision des archives et rapports de fouilles, pour localiser des vestiges architecturaux non publiés qui ont pu être des latrines. Une deuxième étape serait de cartographier les systèmes urbains d'alimentation en eau ainsi que le réseau des rues et des voies pour identifier l'emplacement le plus probable des latrines publiques.

Les latrines sont inestimables pour notre compréhension de l'approvisionnement en eau et des systèmes de drainage d'une ville, du comportement collectif et de la vie quotidienne. Le contenu des égouts associés peut nous informer sur les activités qui ont eu lieu à l'intérieur ou dans leur proche environnement, mais aussi dévoiler les pratiques alimentaires par le biais d'études archéobotaniques, tandis que les analyses

30. Par exemple, A. BouET, Les Latrines dans les provinces gauloises, germainiques et alpines, Paris, 2009 ; B. Hobson, Latrinae et Foricae :Toilets in the Roman World, Londres, 2009 ; G. C. M. Jansen, A. O. Koloski-Ostrow et E. M. Moormann éd., Roman Toilets... 
des sédiments peuvent permettre de découvrir d'anciennes maladies et parasitoses. Ces nouveaux axes de recherche apporteront une profondeur passionnante à la compréhension de la vie urbaine dans le Proche-Orient de l'Antiquité tardive.

Texte traduit de l'anglais par Marie-Odile Rousset

Louise Blanke - Classical Studies, Aarhus Universitet, Danemark

\section{Trois latrines publiques dans la Jérash de l'Antiquité tardive_(Jordanie)}

Cette étude présente trois latrines publiques fouillées à Jérash. Elle examine leur emplacement dans la ville, leur plan ainsi que les systèmes d'adduction et d'évacuation des eaux. Des comparaisons avec des installations trouvées dans d'autres régions du Proche-Orient permettent d'évaluer le nombre d'utilisateurs. L'article met l'accent sur des latrines qui ont été fouillées entre 2008 et 2010 dans le cadre du projet jordano-danois Islamic Jerash Project. Intégrées dans les bains centraux de Jérash, les latrines ont été fréquentées du IVe à la fin du VII e siècle. Elles ont été construites pour l'usage des clients de l'établissement balnéaire, mais pouvaient aussi être utilisées par des nonbaigneurs, qui accédaient alors gratuitement à cette installation. Le mobilier retrouvé dans le comblement de l'égout offre non seulement un aperçu rare des activités ayant lieu dans les bains publics, mais donne aussi une idée de la manière dont l'élimination des déchets pouvait avoir été organisée dans le centre-ville de Jérash.

Antiquité tardive - approvisionnement en eau - bains - Jérash - Jordanie, latrines

\section{Three Public Latrines in Late Antique Jerash (Jordan)}

This study describes three public latrines excavated in Jerash. It examines their location in the city, their layout and also their water supply and drainage systems. Comparisons with facilities found in other parts of the Near East, allow to estimate how many people could have used them. The focus of this study is a latrine that was excavated between 2008 and 2010 as a part of the Danish-Jordanian Islamic Jerash Project. Integrated in the layout of Jerash's Central Bathhouse, the latrine was in use from the 4th to the late 7th century. The latrine was constructed for the use of patrons of the Central Bathhouse, but could also be used by non-bathers, who could access the facilities without paying the fee to enter the bathhouse. The material retrieved from the latrine sewer not only offers a rare insight into activities taking place within the bathhouse, but also gives an insight into how waste disposal could be organised in the centre of the city.

Bathhouse - Jerash - Jordan - Late Antiquity - Latrine - Water Supply 